CERN-TH/99-102

NSF-ITP-99-23

hep-th/9904114

\title{
Stability of Vector Bundles From F-theory
}

\author{
P. Berglund ${ }^{1}$ and P. Mayr 2 \\ ${ }^{1}$ Institute for Theoretical Physics, University of California, Santa Barbara, CA 93106, USA \\ 2 Theory Division, CERN, 1211 Geneva 23, Switzerland
}

\begin{abstract}
We use a recently proposed formulation of stable holomorphic vector bundles $V$ on elliptically fibered Calabi-Yau $n$-fold $Z_{n}$ in terms of toric geometry to describe stability conditions on $V$. Using the toric map $f: W_{n+1} \rightarrow\left(V, Z_{n}\right)$ that identifies dual pairs of F-theory/heterotic duality we show how stability can be related to the existence of holomorphic sections of a certain line bundle that is part of the toric construction.
\end{abstract}

April 1999

1 berglund@itp.ucsb.edu

2 Peter.Mayr@cern.ch 
The heterotic string compactified on Calabi-Yau $n$ fold $Z_{n}$ gives rise to interesting minimal supersymmetric string vacua with a quite realistic spectrum of gauge symmetries and matter fields [1]. The definition of these vacua, involves the specification of a gauge background $V$ with structure group $H \in G_{0}$ embedded in the perturbative heterotic gauge group $G_{0}=E_{8} \times E_{8}$ or $S O(32)$ such that

$$
c_{1}(V)=0 \bmod 2, \quad \lambda(V)=c_{2}(Z)+[W],
$$

where $\lambda$ is the four-dimensional characteristic class of $V$. The second term $[W]$ applies to a generalization of heterotic vacua which include non-perturbative five-branes [2]. Here $[W]$ is a sum of formal four-forms that integrate to one in the transverse direction to the five-branes. Note that the five-branes necessarily have to wrap two-cycles in a 3-fold compactification; such cycles have to be holomorphic in order to preserve supersymmetry [3].

In addition, the connection on $V$ has to satisfy

$$
F_{a b}=F_{\bar{a} \bar{b}}=0, \quad g_{a \bar{b}} F^{a \bar{b}}=0 .
$$

The first equation says that $V$ is holomorphic, while the second equation is interpreted as some stability condition on $V$. Although an existence theorem exists under certain conditions 四, explicit solutions to (2) have been known only in very special constructions. The difficulty to find appropriate backgrounds $V$ has hampered the study of interesting four-dimensional vacua for a long time.

An entirely independent construction of holomorphic, stable vector bundles/ $V$ on Calabi-Yau $n$-folds $Z_{n}$ has been developed in [6] using a certain limit of type IIA strings compactified on a Calabi-Yau $n+1$ fold $W_{n+1}$. In this paper we will obtain a simple stability criterion for $V$ from the geometrical type II setup. The relation of type IIA strings and holomorphic stable vector bundles can be traced back to a simple extension of mirror symmetry in a $K 3 \times T^{2}$ compactification [7], more specifically a discrete symmetry in the moduli space of this compactification that relates geometric deformations of K3 to Wilson lines on the $T^{2}$ factor. In other words, the physics of Wilson lines on $Z_{1}=T^{2}$, is the same as the physics of a type IIA string compactified on a certain limit $\mathcal{W}_{2}$ of an elliptically $\mathrm{K} 3$ manifold $W_{2}$. The relevant type IIA compactification geometry $\mathcal{W}_{2}$ in this limit is the

3 We will loosely call $V$ a bundle in the following, though our construction naturally yields sheaf generalizations of bundles, which provide the relevant set up for heterotic string compactifications [5]. 
local mirror of a Kähler resolution of an $H$ singularity of an elliptically fibered ALE space. Here $H$ is the structure group of the bundle $V$, as before. Fibering this picture, using the adiabatic argument of $[\mathbb{8}]$, one obtains an equivalence between a type IIA compactification on (the geometric limit $\mathcal{W}_{n+1}$ of) an elliptic and K3 fibered Calabi-Yau manifold $W_{n+1}$ and holomorphic stable vector bundles on an elliptically fibered manifold $Z_{n}$. Holomorphic stable bundles $V$ on smooth elliptic fibrations $Z_{n}$ have been first considered in [9] and subsequently in [10].

The construction in [6] has been formulated in terms of toric geometry which for many purposes is the most useful and most general definition for the Calabi-Yau manifolds $\mathcal{W}_{n+1}$ and $Z_{n}$. The toric construction of the pair $\left(V, Z_{n}\right)$ in terms of an $n+1$ dimensional noncompact Calabi-Yau manifold $\mathcal{W}_{n+1}$ works for any structure group $H$ of the bundle. If $H$ fits into the perturbative heterotic gauge group $G_{0}=E_{8} \times E_{8}$ or $S O(32)$, we can interpret $\left(V, Z_{n}\right)$ as a valid perturbative heterotic vacuum. Precisely in this case one can find an embedding of the geometry $\mathcal{W}_{n+1}$ into a compact Calabi-Yau manifold $W_{n+1}$. The statement that type IIA string compactified on a limit of $W_{n+1}$ is equivalent to the heterotic string compactification on $\left(V, Z_{n}\right)$ then establishes F-theory/heterotic duality [11] [12] in the classical limit.

Toric geometry describes a Calabi-Yau $n$-fold $Z_{n}$ in terms of a convex, toric polyhedron $\Delta_{W_{n+1}}^{*}$, which is the convex hull of a set of integral vertices $\nu_{i}^{\star}$ in a standard integral lattice $N$. It is satisfying to observe how the heterotic physics such as the compactification manifold $Z_{n}$ and some defining data of the bundle $V$ have a very simple representation in terms of the polyhedron $\Delta_{W_{n+1}}^{*}$. Moreover also non-perturbative dynamics of the heterotic vacuum for singular configurations, in particular non-perturbative gauge symmetries and non-perturbative five-branes that appear in (1), can be read of from the toric data of $W_{n+1}$ in a simple way. It is the purpose of this note to extend the dictionary between physical and toric data developed in [6] to describe stability of the bundle $V$ in terms of convexity of the polyhedron $\Delta_{W_{n+1}}^{*}$.

In particular it was noted in [6], that for a given structure group $H$ of the bundle, convexity of the toric polyhedron $\Delta_{\mathcal{W}}^{\star}{ }_{3}$ translates to a bound on the first Chern class $\eta=$ $c_{1}(\mathcal{N})$ of a line bundle $\mathcal{N}$ that is an important characteristic of the bundle $V$. Observing that in six dimensions, the bound on $\eta$ from convexity of $\Delta^{\star}$ translates to the stability of instantons on $\mathrm{K} 3$ with structure group $H$, these bounds were interpreted as a similar stability condition in lower dimensions. A general formula for the stability of $V$ in terms of

\footnotetext{
4 See [6] for a list of reviews on toric geometry in the physics literature.
} 
bounds on $\eta$ was subsequently conjectured in ref. [13] which was however not in agreement5 with the original bounds in [6]. In the following we derive a general stability bound for $\eta$ from the toric representation of data $\left(V, Z_{n}\right)$ which is consistent and, we believe, gives a correct treatment.

We start with a sketch of the toric description of the pair $\left(V, Z_{n}\right)$ in terms of the non-compact Calabi-Yau $n+1$-fold $\mathcal{W}_{n+1}$. The latter is elliptically fibered with base $\tilde{B}_{n}$ and ALE fibered with base $B_{n-1}$. The manifold $\mathcal{W}_{n+1}$ is defined as the vanishing locus of a polynomial $p$ in a toric ambient space with coordinates $\left(y, x, \tilde{z}, v, x_{i}\right)$, where $x_{i}$ denote the coordinates of the base $B_{n-1}$ and $(y, x, \tilde{z}, v)$ are certain coordinates on the ALE fiber. The general form of $p$ is

$$
p=p_{0}+p_{+}=p_{0}\left(y, x, \tilde{z} ; x_{i}\right)+\sum_{j=1}^{J} v^{j} p_{+}^{j}\left(y, x, \tilde{z} ; x_{i}\right)
$$

where $p_{0}$ and $p_{+}^{j}$ are quasi-homogeneous polynomials. In particular the $v$ independent piece $p_{0}$ describes an elliptically fibered Calabi-Yau manifold $Z_{n}: p_{0}=0$, while the polynomials $p_{+}^{j}$ contain the information about the bundle $V$. More specifically, each node in the affine Dynkin diagram $\Gamma(H)$ with Dynkin index $s_{i}$ contributes one monomial to the polynomial $p_{+}^{s_{i}}$; in particular $J=\max \left(s_{i}\right)$. E.g. in the case $H=S U(N)$, we have $N$ nodes of index 1 , each of which contributes a monomial to $p_{+}^{1}$. The precise form of the $v$ dependent part of $p$ is $p_{+}=v\left(a_{N}\left(x_{i}\right) \tilde{z}^{N}+a_{N-2}\left(x_{i}\right) \tilde{z}^{N-2} x+\ldots+a_{0}\left(x_{i}\right) x^{N / 2}\right)$ for $N$ even with the last term being $\sim y x^{(N-3) / 2}$ for $N$ odd. The complex parameters multiplying the monomials in $p_{+}$give a projective parametrization of the moduli space of the $S U(N)$ bundle.

Thus the structure group $H$ determines the $y, x, \tilde{z}, v$ content of the monomials appearing in the defining equation $p$ of $\mathcal{W}_{n+1}$. It does not determine the dependence on the base variables $x_{i}$, however. This dependence specifies part the topological class of $V$ that enter the higher Chern classes $c_{2}(V), c_{3}(V), \ldots$. However note that this dependence is quite restricted because of the quasi-homogeneousness of $p$. It was shown in [6] that the dependence of the monomials in $p$ on $x_{i}$ is completely determined by specifying the action of two $C^{*}$ actions acting on $\left(y, x, \tilde{z}, v, x_{i}\right)$. In other words, we have to specify two line bundles $\mathcal{L}$ and $\mathcal{M}$ on the base $B_{n-1}$ and the coordinates $\left(y, x, \tilde{z}, v, x_{i}\right)$ transform as certain sections of $\mathcal{M}$ and $\mathcal{L}$ such that

$$
y \sim \mathcal{M}^{3} \mathcal{L}^{3}, \quad x \sim \mathcal{M}^{2} \mathcal{L}^{2}, \quad \tilde{z} \sim \mathcal{M}, \quad v \sim \mathcal{M}^{5-N}, \quad f_{c, d} \sim \mathcal{M}^{d} \mathcal{L}^{c}
$$

5 The discriminant criterion used in 13 is not restrictive enough to fix the structure group $H$. 
where $f_{c, d}=f_{c, d}\left(x_{i}\right)$ is the base dependent part in a monomial

$$
y^{(6-2 b-c) / 3} x^{b} \tilde{z}^{c} v^{d} f_{c, d}\left(x_{i}\right)
$$

of $p$. Moreover $N$ is the highest power of $\tilde{z}$ that appears in $p_{+}^{1}$.

From the fact that $p_{0}=0$ describes a Calabi-Yau manifold it follows that the bundle $\mathcal{L}$ is actually the anti-canonical bundle of $B_{n-1}$. On the other hand $\mathcal{M}$ is an intrinsic property of $V$ alone. In the following we will derive a bound on the first Chern class $c_{1}(\mathcal{N})$ of the line bundle $\mathcal{N}=\mathcal{M} \mathcal{L}^{6}$ from convexity of the polyhedron $\Delta_{W_{n+1}}^{*}$. The change from $\mathcal{M}$ to $\mathcal{N}$ is intended to make contact with the notation of ref. [9], where $V$ has been described in terms of sections of a certain weighted projective bundle $\mathcal{W}$ on $B_{n-1}$ with coordinates on $\mathcal{W}$ being sections of $\mathcal{N}^{s_{i}} \mathcal{L}^{-d_{j}}$. Here $s_{i}$ are the Dynkin indices as above and $d_{i}$ are the degrees of the independent Casimir invariants of $H$. The dependence of higher Chern classes of $V$ on $c_{1}(\mathcal{N})$ has been determined in [9] [14].

To keep the discussion as basic as possible we will work directly with the defining polynomial $p$ rather than with the toric polyhedron $\Delta_{\mathcal{W}_{n+1}}^{\star}$ that determines $p$ [15]. In particular, if we consider a structure group $H \in G_{0}$, we have a compact embedding $\mathcal{W}_{n+1} \rightarrow W_{n+1}$. Moreover the K3 fiber (that, in a local patch, contains the ALE fiber of $\mathcal{W}_{n+1}$ ) has a singularity $G$ which is the commutant of $H$ in $G_{0}$ [12]. The defining polynomial $\hat{p}$ of $W_{n+1}$ can be written in generalized Weierstrass form:

$$
\hat{p}=y^{2}+x^{3}+y x \hat{z} a_{1}+x^{2} \hat{z}^{2} a_{2}+y \hat{z}^{3} a_{3}+x \hat{z}^{4} a_{4}+\hat{z}^{6} a_{6}
$$

Note that we use $(y, x, \hat{z})$ and $(y, x, \tilde{z})$ to denote the homogeneous coordinates of the elliptic fiber of the $n+1$-dimensional Calabi-Yau $W_{n+1}$ and the $n$ dimensional Calabi-Yau $Z_{n}$, respectively. The $a_{n}$ are functions of the coordinates $\tilde{x}_{i}$ of the base $\tilde{B}_{n}$ of the elliptic fibration of $W_{n+1}$. In particular having a singularity of type $G$ above a locus, say at $z \equiv \tilde{x}_{1}=0$ on the base $\tilde{B}_{n}$ can be phrased in terms of the behavior of the $a_{n}$ near $z=0$, $a_{n} \sim z^{\delta_{n}}$ using Tate's algorithm [16].

To reiterate, the structure group $H$ determines the singularity $G$ of the K3 fiber of $W_{n+1}$. In turn $G$ is determined by the behavior of the coefficient functions $a_{n}$ in the generalized Weierstrass form $\hat{p}$ near the singularity $z=0$. Moreover we are interested in the singularity of the K3 fiber $W_{2}$ of the K3 fibration $W_{2} \rightarrow W_{n+1} \rightarrow B_{n-1}$. Thus $z$ is now a coordinate on the base $\mathbf{P}^{1}$ of the elliptically fiberered K3, $\pi: W_{2} \rightarrow \mathbf{P}^{1}$. However note that in the local limit $W_{n+1} \rightarrow \mathcal{W}_{n+1}$ where our map $f: \mathcal{W}_{n+1} \rightarrow\left(V, Z_{n}\right)$ applies, the $a_{n}$ 
become nothing but the $\tilde{B}_{n}$ dependent parts of the monomials $y^{(6-b-c) / 3} x^{b} \tilde{z}^{c} v^{d} f_{c, d}\left(x_{i}\right)$ in the defining polynomial $p$ of $\mathcal{W}_{n+1}$.

To be more explicit let us consider the case $G_{0}=E_{8} \times E_{8}$. The relation between the coordinates in $\hat{p}$ and $p$, using $z \equiv \tilde{x}_{1}, w \equiv \tilde{x}_{2}$ as the coordinates of the base $\mathbf{P}^{1}$ of $W_{2}$ as above, is then

$$
\left(y, x, \tilde{z} ; v ; x_{i}\right)_{p}=\left(y, x, \hat{z} z w ; w / z ; \tilde{x}_{i}, i>3\right)_{\hat{p}} .
$$

In particular the existence of a term $a_{n}=z^{\delta_{n}} f\left(x_{i}\right)$ implies that the line bundle

$$
\mathcal{N}^{d_{n}} \mathcal{L}^{n-6 d_{n}}
$$

has a holomophic section, where we have defined $d_{n}=n-\delta_{n}$. Combining the conditions imposed by all the $a_{n}, n \in\{1,2,3,4,6\}$ we arrive at the condition

$$
\nu(G) c_{1}(\mathcal{L}) \leq \eta \quad\left(\leq 12 c_{1}(\mathcal{L})\right),
$$

where $\nu(G)$ is the singularity dependent quantity

$$
\nu(G)=\max \left(\frac{\left(6 d_{n}-n\right)}{d_{n}}\right),
$$

for all $n \in\{1,2,3,4,6\}$ with $d_{n} \neq 0$. Note that this argument also tells us that the minimal bundle $\mathcal{N}_{\text {min }}$ is always a power of $\mathcal{L}$, a fact that is non-trivial for $h^{1,1}(B)>1$. We have also indicated in (7) an upper bound for $\eta$ of a very different origin. It is not related to the stability of $\mathcal{N}$ but rather to the stability of $\eta^{\prime}=c_{1}\left(\mathcal{N}^{\prime}\right) \geq 0$, where $\mathcal{N}^{\prime}$ is the equivalent bundle in the second $E_{8}$ factor.

From eqs. (7),(8) we obtain

$$
\begin{array}{llll}
G=S U(2)^{*}: & \eta \geq \frac{14}{3} \cdot L & G=E_{8}: & \eta \geq 0 \cdot L \\
G=S U(3)^{*}: & \eta \geq \frac{9}{2} \cdot L & G=E_{7}: & \eta \geq 2 \cdot L \\
G=S U(M): & \eta \geq 5 \cdot L & G=E_{6}: \quad \eta \geq 3 \cdot L \\
G=S O(N): & \eta \geq 4 \cdot L & G=G_{2}: \quad \eta \geq 4 \cdot L \\
& & G=F_{4}: \quad \eta \geq 3 \cdot L
\end{array}
$$

Here $N \in\{7, \ldots, 12\}, M \in\{2, \ldots, 6\}$ and the groups with a star denote the singularities associated to $I I I$ and $I V^{s}$ fibers in the list of [16]. Moreover $L=c_{1}(\mathcal{L})$. Note that our arguments for the bound (7) give a necessary but in general not sufficient criterion for the stability of the bundle. In particular the distinction between singularities associated to simply laced (SL) and non-simply laced (NSL) groups is often the specific form of the 
sections $f_{c, d}$ in the SL case rather than a difference in the topological class [16]. Our arguments give a criterion for the existence of a holomorphic section $f_{c, d}$ which is sufficient for SL groups, but it does not ensure that one gets a generic enough section to describe also the NSL case. Thus the bounds given in (9) are strict lower bounds only for the simply laced case.

It would be nice to have a derivation of the bound on $\eta$ that is directly related to the structure group $H$ and in particular also applies to the non-compact case with arbitrary rank. A reasonable proposal based on (9) is

$$
\begin{aligned}
& H=S U(N): \quad \eta \geq N \cdot L \quad H=E_{8}: \quad \eta \geq 5 \cdot L \\
& H=S O(7): \quad \eta \geq 4 \cdot L \quad H=E_{7}: \quad \eta \geq \frac{14}{3} \cdot L \\
& H=S O(M): \quad \eta \geq \frac{M}{2} \cdot L \quad H=E_{6}: \quad \eta \geq \frac{9}{2} \cdot L \\
& H=S p(K): \quad \eta \geq 2 K \cdot L \quad H=G_{2}: \quad \eta \geq \frac{7}{2} \cdot L \\
& H=F_{4}: \quad \eta \geq \frac{13}{3} \cdot L,
\end{aligned}
$$

where $N \geq 2, M \geq 8$ and $K \geq 2$. Here we have used the calculation of the six-dimensional spectrum in Table 3 of [16] to raise the bounds for the NSL cases, such that we get the correct answer in six dimensions. Similarly we could compare the calculation of the matter spectrum in four dimension for a heterotic compactification on a Calabi-Yau 3-fold with (9).

It is interesting to observe, how the geometric type IIA description contains non-trivial information about the instanton dynamics on Calabi-Yau manifolds $Z_{n}$. Moreover in many cases there is also another point of view, namely in terms of a supersymmetric field theory in uncompactified space time. In particular, via the heterotic compactification on $K 3 \times T^{2}$, the instanton dynamics on $\mathrm{K} 3$ is related to the moduli space of $\mathcal{N}=2$ supersymmetric field theories. Similarly, instantons on Calabi-Yau three-folds will be related to the moduli space of $\mathcal{N}=1$ Super-Yang-Mills theories. It would be very interesting to use the toric type IIA construction of $V$ on $Z_{n}$ to study this relation in detail.

\section{Acknowledgements:}

P.M. would like to thank Govindan Rajesh for discussions. P.B. was supported in part by the Natural Science Foundation under Grant No. PHY94-07194. P.B. would also like to acknowledge LBL, Berkeley for hospitality during the course of this work. 


\section{References}

[1] P. Candelas, G.T. Horowitz, A. Strominger and E. Witten, Nucl. Phys. $\underline{\text { B258 }}$ (1985) 46 ;

C. Hull and E. Witten, Phys. Lett. $\underline{B 160}$ (1985) 398.;

E. Witten, Nucl. Phys. $\underline{B 268}$ (1986) 79;

J. Distler, Phys. Lett. $\underline{B 188}$ (1987) 431;

J. Distler and B. Greene, Nucl. Phys. B304 (1988) 1.

[2] M.J. Duff, R. Minasian and E. Witten, Nucl. Phys. B465 (1996) 413.

[3] K. Becker, M. Becker and A. Strominger, Nucl. Phys. B456 (1995) 130;

M. Bershadsky, V. Sadov and C. Vafa, Nucl. Phys. B463 (1996) 420.

[4] K. Uhlenbeck and S.T. Yau, On the existence of hermitian Yang-Mills connections in stable vector bundles, preprint (1986).

[5] J. Distler, B.R. Greene and D.R. Morrison, Nucl. Phys. B481 (1996) 289.

[6] P. Berglund and P. Mayr, Heterotic string/F-theory duality from mirror symmetry, hep-th/9811217.

[7] S. Katz, P. Mayr and C. Vafa, Adv. Theor. Math. Phys. 1 (1998) 53.

[8] C. Vafa and E. Witten, Nucl. Phys. Proc. Suppl. $\underline{46}$ (1996) 225.

[9] R. Friedman, J.W. Morgan and E. Witten, Comm. Math. Phys. 187 (1997) 679.

[10] M. Bershadsky, A. Johansen, T. Pantev and V. Sadov, Nucl. Phys. $\underline{B 505}$ (1997) 165;

R.Y. Donagi, Asian J. Math. 1 (1997) 214; MSRI pub. 28 (1992) 65;

G. Curio and R.Y. Donagi, Nucl. Phys. $\underline{B 518}$ (1998) 603.

[11] C. Vafa, Nucl. Phys. B469 (1996) 403

[12] C. Vafa and D. Morrison, Nucl. Phys. $\underline{B 473}$ (1996) 74; Nucl. Phys. $\underline{B 476}$ (1996) 437.

[13] G. Rajesh, Toric geometry and F theory / heterotic duality in four-dimensions, hepth/9811240.

[14] B. Andreas, On vector bundles and chiral matter in $N=1$ heterotic compactifications, hep-th/9802202; G. Curio, Phys. Lett. B435 (1998) 39.

[15] V. Batyrev, Duke Math. Journ. $\underline{69}$ (1993) 349.

[16] M. Bershadsky et al., Nucl. Phys. Bu81 (1996) 215. 